\title{
GRASPing Examination Board Assignments for University-Entrance Exams
}

\author{
Jorge Puente ${ }^{1}$, Camino R. Vela ${ }^{1}$, Inés González-Rodríguez ${ }^{2}$, \\ Luis J. Rodríguez ${ }^{3}$, and Juan José Palacios ${ }^{1}$ \\ 1 A.I. Centre and Department of Computer Science, \\ University of Oviedo, (Spain) \{puente, crvela, palaciosjuan\}@uniovi.es, \\ 2 Department of Mathematics, Statistics and Computing, \\ University of Cantabria, (Spain) ines.gonzalez@unican.es, \\ 3 Department of Statistics and O.R., \\ University of Oviedo, (Spain), luisj@uniovi.es.
}

\begin{abstract}
In the sequel, we tackle a real-world problem: forming examination boards for the university-entrance exams at the autonomous region of Asturias (Spain). We formulate the problem, propose a heuristic GRASP-like method to solve it and show some experimental results to support our proposal.
\end{abstract}

\section{Introduction}

In Spain, students who finish Secondary School must take a University Entrance Exam (UEE), consisting of several modules or subjects (Maths, Chemistry, Art...), some of which are optional. The mark obtained in this exam is key for entering university. It is therefore the responsibility of the organisers to provide the best possible environment for the examination process. Its organisation is undertaken independently in each of the 17 Spanish autonomous regions by an Organising Committee, and it includes deciding on aspects such as the number of days allocated to the exam, the number and location of exam venues or the optional exam-modules offered in each venue.

A critical issue for the committee is to decide for each venue on the composition of an examination board in charge of invigilation. Exam venues with varying sizes require a different number of examiners in order to provide proper invigilation. It is also necessary that examiners cover different areas of expertise, so they can adequately reply to students' questions. While the board's composition must guarantee high standards in terms of expertise and invigilation capacity, examiners' travelling expenses incur an economical cost which should be kept to a minimum.

In the following, we shall tackle the Examination Board Assignment (EBA) problem. After modelling the problem in Section 2, we shall propose in Section 3 a GRASP algorithm to solve it. To evaluate our proposal, in Section 4 we will present some detailed results analysing the different parts of the algorithm as well as compare it with the solution provided by the human experts on some real-world instances of the problem. Finally, Section 5 presents some conclusions. 


\section{Problem Definition and Formulation}

The Organising Committee in the Principality of Asturias (the region where the University of Oviedo lies) is in charge of a varying number of UEE examination venues geographically distributed throughout the region's territory. Students are assigned to a specific venue depending on their school of origin. The comittee takes into account the total number of students registered for that particular UEE session as well as the optional modules they intend to take to select a set of teachers (examiners hereafter) for invigilation. These examiners can be university staff or secondary-school teachers (these include education supervisors, on special duty at the Education Department of the Administration). Given each examiner's academic profile, he/she is associated to a particular subject and is also meant to have different levels of affinity to every other subject, which make him/her more or less adequate to answer student questions during the examination process. The Committee appoints for each board a president and a secretary, who must be university academic staff and imposes some additional constraints on the composition of the boards, including their size. The goal in the EBA is to assign all the examiners from the initial set to different examination boards given the predefined assignment of president and secretary, in such a way that all constraints hold and the following objectives are optimised:

1. It is not always possible to assign an expert on each subject to every board; instead, the affinity concept is used and the objective is to maximise the expertise affinity coverage provided by the examiners on each board.

2. Examiners get paid for their travel expenses if they are assigned to a venue located elsewhere than their usual workplace. The total amount depends on the distance and may include accommodation expenses. Clearly, travel costs should be minimised.

Having outlined the problem statement, we give some more detail about its different components in the following.

The input data available when organising each UEE session are the following:

1. Examiners Let $E$ denote the set of examiners. For each examiner $e \in E$, we are given the following information: subject in which he/she is specialised, denoted $\operatorname{Subj}(e)$; type of examiner, which can be university lecturer, education supervisor or secondary school teacher on active duty; school, if he/she is a secondary school teacher, denoted $\operatorname{School}(e)$; and city or town where his/her workplace is located, denoted $\operatorname{Town}(e)$.

2. Venues Let $V$ denote the set of venues. For each venue $v \in V$ we are given the following information: preassigned president and secretary from $E$; set of the secondary schools associated to this venue, denoted Lschools $(v)$; set of subjects for which exams will take place at this venue, denoted $\operatorname{Lsubj}(v)$; location (city or town), denoted Location $(v)$; and size of the examination board (i.e. number of examiners) for that venue, denoted $\operatorname{Size}(v)$. It is assumed that $\sum_{v \in V} \operatorname{Size}(v)=|E|$. 
3. Subjects Let $S$ be the set of subjects where each subject $s \in S$ is characterised by a textual description.

4. Affinity array, where rows correspond to examiners and columns to subjects, so $A(e, s) \in[0,1]$ is the affinity degree between examiner $e$ and subject $s$, elicited by the Organising Commitee based on the area of expertise of each examiner so it usually corresponds to the affinity between $\operatorname{Subj}(e)$ and $s$.

5. Data related to travelling costs, namely: table with distances between different towns or cities where exam venues, schools or university campus may be located, so distance $(a, b)$ denotes the distance between locations $a$ and $b$; travelling cost per kilometre $\left(c_{k m}\right)$; distance threshold for staying overnight and claiming accommodation expenses $(\Delta)$; accommodation cost per night $(h)$; travelling allowance per day $(a)$; and duration (in days) of the exam $\left(n_{d}\right)$.

The problem solution is the Examination Board Assignment (EBA for short), specifying the set Board $(v)$ of examiners composing the board for each venue $v \in V$. This set is the disjoint union of the sets of university and secondaryschool teachers, denoted $\operatorname{UBoard}(v)$ and $\operatorname{SBoard}(v)$ respectively. The latter includes both secondary-school teachers on active service and education supervisors, that is, there is a subset $\operatorname{ESBoard}(v) \subset \operatorname{SBoard}(v)$ formed by those examiners in $\operatorname{Board}(v)$ on special duty as education supervisors. The solution must also provide the following information for each venue $v \in V$ : the total cost associated to its board, denoted $T C(v)$, and the degree of coverage for each subject $s \in \operatorname{Lsubj}(v)$, denoted Coverage $(v, s)$, as well as the overall coverage $A C(v)$.

Additional requirements of the Organising Committee translate into five constraints for this problem; the first two are hard, while the remaining ones are soft and may be relaxed if needed.

1. The predefined and final size of each exam board must coincide:

$$
C_{1}: \forall v \in V|\operatorname{Board}(v)|=\operatorname{Size}(v)
$$

where $|\operatorname{Board}(v)|$ denotes the cardinality of set Board $(v)$.

2. Teachers from secondary schools associated to a venue may not be assigned to it (since their students are taking the exam in that same venue):

$$
C_{2}: \forall v \in V \forall e \in \operatorname{SBoard}(v) \operatorname{School}(e) \notin \operatorname{Lschools}(v) .
$$

3. It is preferred that an equilibrium exists between the number of university lecturers and secondary-school teachers in each venue:

$$
C_{3}: \forall v \in V \frac{|\operatorname{UBoard}(v)|}{|\operatorname{Board}(v)|} \in[0.4,0.6] .
$$

4. The number of venues where a subject is taken and the number of examiners expert on that subject may differ substantially. To distribute experts as 
homogenously as possible, an upper bound is established for each each venue $v \in V$ and subject $s \in \operatorname{Lsubj}(v)$ as follows:

$$
M(v, s)=\left\lceil 1+|\{e \in E: s=\operatorname{Subj}(e)\}| * \frac{\operatorname{Size}(v)}{\sum_{v^{\prime} \in V: s \in \operatorname{Lsubj}\left(v^{\prime}\right)} \operatorname{Size}\left(v^{\prime}\right)}\right\rceil
$$

where $\lceil x\rceil$ denotes the nearest integer greater than or equal to $x$. The constraint is then expressed as:

$$
C_{4}: \forall v \in V, \forall s \in \operatorname{Lsubj}(v)|\{e \in \operatorname{Board}(v): s=\operatorname{Subj}(e)\}| \leq M(v, s) .
$$

5 . It is preferred that education supervisors are equally distributed across all venues:

$$
C_{5}: \max _{v \in V}|\{e \in \operatorname{ESBoard}(v)\}|-\min _{v \in V}|\{e \in \operatorname{ESBoard}(\mathrm{v})\}| \leq 1 .
$$

Regarding the objectives, the most important one is to maximise the degree of expertise coverage provided by the members of each board. For any venue $v \in S$ and any subject $s \in \operatorname{Lsubj}(v)$, the degree of coverage of $s$ at $v$ is given by Coverage $(v, s)=\max _{e \in \operatorname{Board}(v)}\{A(e, s)\}$ and the overall coverage at a venue $v \in V$ is calculated as:

$$
A C(v)=\frac{\sum_{s \in \operatorname{Lsubj}(v)} \operatorname{Coverage}(v, s)}{|\operatorname{Lsubj}(v)|}
$$

Notice that $A C(v) \in[0,1]$ for every venue $v \in V$, with $A C(v)=1$ meaning a perfect coverage. We can then formalise the first objective as:

$$
\min f_{1}=|V|-\sum_{v \in V} A C(v) .
$$

Regarding the second objective, the cost of assigning an examiner $e \in E$ to a venue $v \in V$ is computed as:

$$
\operatorname{Cost}(e, v)= \begin{cases}0 & \text { if } d_{v e}=0, \\ 2 d_{v e} c_{k m} n_{d}+a n_{d} & \text { if } 0<d_{v e} \leq \Delta, \\ 2 d_{v e} c_{k m} n_{d}+a n_{d}+\left(n_{d}-1\right) h & \text { if } d_{v e}>\Delta,\end{cases}
$$

where $d_{v e}=$ distance(Location $\left.(v), \operatorname{Town}(e)\right)$ is the distance between the location of venue $v$ and the workplace of examiner $e$. The overall cost for a venue $v \in V$ is then given by $T C(v)=\sum_{e \in \operatorname{Board}(v)} \operatorname{Cost}(e, v)$ so the second objective is:

$$
\min f_{2}=\sum_{v \in V} T C(v)
$$

Since the most important objective for the Organising Committee is to provide a good expertise coverage, we adopt a lexicographic approach [1] and define the examination board assignment problem as follows:

$$
\begin{aligned}
& \operatorname{lexmin}\left(f_{1}, f_{2}\right) \\
& \text { subject to: } C_{i}, 1 \leq i \leq 5 .
\end{aligned}
$$


where lexmin denotes lexicographically minimising the vector $\left(f_{1}, f_{2}\right)$ : if $f(S)$ denotes the value of objective $f$ for a feasible solution $S, S$ is preferred to $S^{\prime}$, denoted $s \preceq_{\text {lexmin }} s^{\prime}$, iff $f_{1}(S)<f_{1}\left(S^{\prime}\right)$ or $f_{1}(S)=f_{1}\left(S^{\prime}\right)$ and $f_{2}(S) \leq f_{2}\left(S^{\prime}\right)$. Lexicographic minimisation is well-suited to seek a compromise between conflicting interests, as well as reconciling this requirement with the crucial notion of Pareto-optimality [2].

The problem above is related to personnel scheduling problems [3],[4]. It shares with this family of problems objective functions and constraints, for example, coverage constraints, which are among the most important soft constraints [4]. However, it is not a typical problem from this family: we do not need to schedule shifts or days off, there is no single workplace (as in nurse rostering), all "tasks" in our problem demand "specific skills" and despite having groups of employees, these have individual profiles. All in all, the specific characteristics of the problem under consideration allow for no comparisons with other proposals from the literature. Finally, it is possible to prove that the resulting problem is $N P$-hard (we omit the proof due to lack of space).

\section{Solving the Problem with a GRASP-like Algorithm}

The complexity of the problem under consideration suggests using meta-heuristic methods to solve it. Such methods allow problem-specific information to be incorporated and exploited, as well as making it easy to deal with complex objectives, in particular with "messy real world objectives and constraints" [3]. Here, we propose a solving method inspired in GRASP (Greedy Randomized Adaptive Search Procedure) meta-heuristics [5]. GRASP methods have proved popular for solving personnel scheduling problems (see [6] and references therein). This is in part due to the fact that these problems can be modelled as allocation problems and neighbourhood structures can be naturally defined by moving a single allocation or swapping two or more allocations. It is also natural to fix one or more allocations at each step of the construction phase.

Our method, in Algorithm 1, consists of a construction phase followed by an improvement phase. The construction phase is in itself divided in two steps: first, a Sequential-Greedy-Randomized-Construction algorithm (SGRC) builds an initial solution and then, if this solution is not feasible, a Solution-Repair algorithm (SR) obtains a feasible one by relaxing some of the soft constraints. Once a complete solution is available, the second phase performs a Local Search (LS) where the solution's neighbourhood is systematically explored until a local optimum is found. As random decisions are present in every phase, the process is repeated NRestarts times. The output is the best solution from all runs chosen as follows: for a pair of feasible solutions, we use $\preceq_{\text {lexmin }}$; else, feasible solutions are preferred to unfeasible ones and, between two unfeasible solutions, preference is established in terms of objective values as well as constraint satisfaction. 


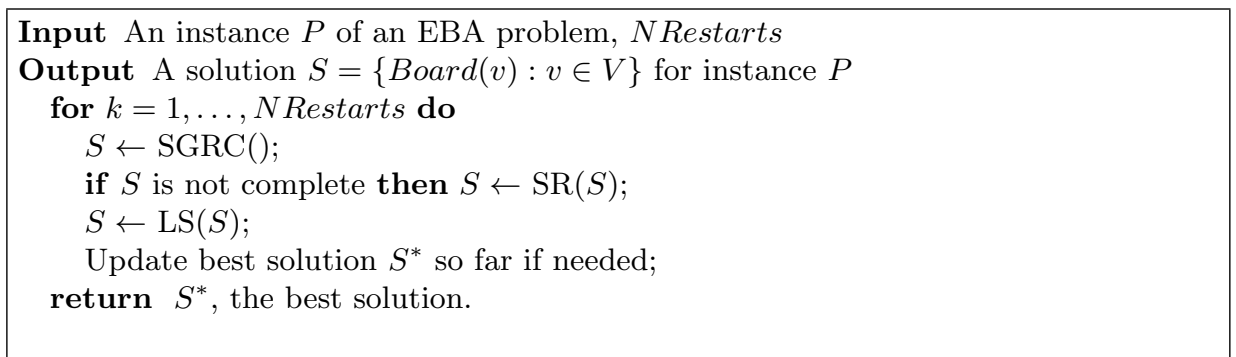

Algorithm 1: GRASP-like algorithm for the EBA problem

\subsection{Sequential Greedy Randomized Construction}

The SGRC starts by assigning the president and the secretary to each board as specified in the input data. Then, examiners from $E$ not assigned yet are allocated to boards in tree consecutive steps: assignment of education supervisors, assignment of "covering" examiners and assignment of "non-covering" examiners. Non-determinism situations are in some cases solved using heuristic criteria and in others introducing randomness, thus increasing the diversity of the greedy algorithm. In all cases, all constraints must be complied with, so it is possible that, at the end of SGRC some examiners from $E$ still remained unassigned. In this case, SGRC yields a partial solution that needs to be repaired.

In the first step, provided that $C_{5}$ holds, each education supervisor is assigned to that venue $v$ where its contribution to the coverage of $\operatorname{Board}(v)$ is maximum. Ties between venues are broken using costs. In the second step, venues are sorted in increasing-size order and, following that order, for each venue, valid examiners are assigned to it as long as the venue's board is not full and the examiner's contribution to the venue's coverage is positive and maximal. An examiner is valid if he/she satisfies constraints $C_{2}$ and $C_{4}$ as well as two partial constraints $C_{1}^{\prime}:|\operatorname{Board}(v)| \leq \operatorname{Size}(v)$ and $C_{3}^{\prime}:|\operatorname{BBoard}(v)|,|\operatorname{SBoard}(v)| \leq 0.6 \operatorname{Size}(v)$ (satisfying $C_{1}^{\prime}$ and $C_{3}^{\prime}$ along SGRC means that $C_{1}$ and $C_{3}$ will be satisfied in the end). Ties are broken at random. At the end of the second step, there may be examiners in $E$ not assigned because of their null contribution to the covering of compatible boards. In the third step those non-covering examiners are assigned to venues (ordered at random) keeping costs to their minimum.

\subsection{Solution Repair}

SGRC may end without assigning all the examiners in $E$ because doing so would violate some constraints. In this case, SR builds a full solution by relaxing some of the soft constraints, with a possible deterioration in the coverage objective.

First, $C_{4}$ is relaxed in as few venues as possible, this being the constraint that has empirically proved to be more conflicting. For each venue $v$ with free positions in its board we pick a non-assigned examiner $e$ at random. Then we consider all pairs $\left(e^{\prime}, v^{\prime}\right)$ such that either $v^{\prime}=v, e^{\prime}=e$ or $v^{\prime} \neq v, e^{\prime} \in \operatorname{Board}\left(v^{\prime}\right)$ and such that assigning $e$ to $\operatorname{Board}\left(v^{\prime}\right)-\left\{e^{\prime}\right\}$ and $e^{\prime}$ to $\operatorname{Board}(v)$ satisfies constraints 
$C_{1}^{\prime}, C_{2}, C_{3}^{\prime}$ and $C_{5}$. If more than one of such pairs exist, we select the pair maximising $A C(v)+A C\left(v^{\prime}\right)$ after the "swap" and exchange the examiners. This procedure continues until Board $(v)$ is complete or no swaps are possible. In the case that relaxing $C_{4}$ were not enough, we would start all over again asking for the swap to satisfy only $C_{1}^{\prime}$ and $C_{2}$ (i.e. we relax the remaining soft constraints simultaneously). It is possible to prove that this process always terminates with a full solution where all venues are complete and all examiners have been assigned.

\subsection{Local Search}

The LS starts from the full solution provided by SRGC+SR and explores its neighbourhood trying to improve on objective values and constraint satisfaction. To this end, it considers the list, ordered by venue, of examiners who are not education supervisors nor a prefixed president or secretary. Then, the neighbourhood is generated using a Forward Pairwise Interchange (FPE) [7]: for each examiner $e$, we consider exchanging it with another examiner $e^{\prime}$ in another board following $e$ in the list; if the reassignment improves the solution, the exchange is made, otherwise, it is discarded. After trying all exchanges, the process starts again from the first examiner in the list. This continues until no exchange has been made for a whole round of trials.

In this process, the degree to which a solution $S$ does not satisfy $C_{4}$ is:

$$
D_{n s}\left(S, C_{4}\right)=\sum_{v \in V} \sum_{s \in \operatorname{LSubjects}(\mathrm{v})}|\{e \in \operatorname{Board}(v): s=\operatorname{Subj}(e)\}|-M(v, s)
$$

Analogously, the degree of dissatisfaction of $C_{3}, D_{n s}\left(S, C_{3}\right)$, is the number of venues $v$ where the equilibrium between university and secondary-school teachers is broken, and the degree of dissatisfaction of $C_{5}, D_{n s}\left(S, C_{5}\right)$, is the number of venues where the number of supervisors does not correspond to a uniform distribution. Then, a solution $S$ is better than or preferred to another solution $S^{\prime}$ (denoted $S \preceq_{L S} S^{\prime}$ ) in the sense of Pareto-dominance [8], that is, iff $f_{1}(S) \leq$ $f_{1}\left(S^{\prime}\right), f_{2}(S) \leq f_{2}\left(S^{\prime}\right), D_{n s}\left(S, C_{i}\right) \leq D_{n s}\left(S^{\prime}, C_{i}\right)$ for $i=3,4,5$ and at least one of these inequalities is strict.

\section{Experimental Results}

To evaluate the proposed algorithm, we shall use three real instances of the EBA problem, corresponding to UEE celebrated in June 2010, 2011 and 2012. Unfortunately, the historical data available before 2010 cannot be used, because the UEE format was different and so was the problem definition.

First, we shall analyse the behaviour of the two main modules of the algorithm, the constructive phase (including repair) and the local search. Then, we will compare the solutions of our algorithm to the solutions provided by the human experts in years 2010 and 2011; in year 2012 the actual solution has already been obtained with the proposed GRASP algorithm and, therefore, no experts' solution is available. 
Table 1. Evaluation of the stages of the proposed GRASP

\begin{tabular}{llcccccc}
\hline Instance & & \multicolumn{2}{c}{ Best } & \multicolumn{2}{c}{ Average } & \multicolumn{2}{c}{ Std. Dev. } \\
& & $A C(\%)$ & $T C(€)$ & $A C(\%)$ & $T C(€)$ & $A C$ & $T C$ \\
\hline \multirow{2}{*}{ June2010 } & SGRC+SR & 74.80 & 38444 & 73.16 & 36209 & 0.0054 & 599.5 \\
& GRASP & 77.46 & 27824 & 77.20 & 28600 & 0.0011 & 451.9 \\
\multirow{2}{*}{ June2011 } & SGRC+SR & 70.31 & 37067 & 68.97 & 34644 & 0.0045 & 669.4 \\
& GRASP & 71.56 & 27846 & 71.32 & 27837 & 0.0009 & 672.9 \\
\multirow{2}{*}{ June2012 } & SGRC+SR & 73.25 & 34087 & 71.80 & 33629 & 0.0042 & 648.38 \\
& GRASP & 75.27 & 26281 & 74.96 & 26372 & 0.0011 & 585.23 \\
\hline
\end{tabular}

The prototype is programmed in $\mathrm{C}++$ on a Xeon E5520 processor running Linux (SL 6.0). In all cases, the number of restarts for the GRASP algorithm is $N$ Restarts $=50$. Also, given its strong stochastic nature, the algorithm is run 50 times to obtain statistically significant results. The average runtime for one run is $391.2,292.8$ and 386.46 seconds for 2010,2011 and 2012 respectively.

\subsection{Analysis of the Algorithm}

The first set of experiments provides a better insight into the algorithm's behaviour, namely, into the constructive phase - denoted SGRC+SR and consisting of SGRC followed by SR - and the local search phase. Since SGRC+SR has $N$ Restarts $=50$ restarts in each run of the GRASP algorithm and we consider results of 50 runs, SGRC+SR is executed 2500 times for fair comparisons.

A summary of the results is as follows: at the end of the first step, in average $70 \%$ of the examiners from $E$ have already been allocated to a venue (between $66 \%$ and $77 \%$ ). At the end of the second step, between 93 and $100 \%$ (97.8\% in average) of the members of $E$ have already been assigned. When solution repair is needed, all pending examiners are assigned after relaxing constraint $C_{4}$, so $C_{3}$ and $C_{5}$ need not be relaxed. Once $\mathrm{SR}$ is finished, the percentage of solutions not satisfying $C_{4}$ is, respectively, 82\%, 89\% and 95\% for years 2010, 2011 and 2012 . Also, the value of $f_{1}$ (expertise covering) remains unchanged during SR.

We now evaluate the contribution of LS to the GRASP algorithm, by comparing the results obtained after the constructive phase (SGRC+SR) (the first set of complete solutions) to those obtained at the end of the GRASP algorithm, after applying local search. Table 1 contains the best, average and standard deviation of the two objective values Average Coverage $(A C)$ and Total Cost of travel expenses $(T C)$ obtained in both cases. It does not report data related to constraint satisfaction because LS always yields solutions where all 5 constraints hold. We can see that LS noticeably reduces the total cost as well as improving the expertise coverage. Clearly, the greatest improvement is in costs, since this objective received considerably less attention in the constructive phase. Costs are reduced in average $9215 €$ for the best solution and $7224 €$ for the average solution. Regarding the expertise coverage, it is not surprising that local search 
Table 2. Comparison between expert and GRASP results

\begin{tabular}{llccccccc}
\hline & & \multicolumn{4}{c}{ Problem Objectives } & \multicolumn{5}{c}{ Constraint satisfaction degree(\%) } \\
Instance & Method & $A C(\%)$ & $T C(e)$ & $C_{1}$ & $C_{2}$ & $C_{3}$ & $C_{4}$ & $C_{5}$ \\
\hline \multirow{2}{*}{ June2010 } & Expert & 73.04 & 32032 & 100 & 100 & 60.00 & 93.40 & 80 \\
& GRASP Best & 77.46 & 27824 & 100 & 100 & 100 & 100 & 100 \\
& GRASP Avg & 77.20 & 28600 & 100 & 100 & 100 & 100 & 100 \\
\multirow{3}{*}{ June2011 } & Expert & 68.20 & 32748 & 100 & 100 & 69.23 & 100 & 61.53 \\
& GRASP Best & 71.56 & 27846 & 100 & 100 & 100 & 100 & 100 \\
& GRASP Avg & 71.32 & 27837 & 100 & 100 & 100 & 100 & 100 \\
\hline
\end{tabular}

makes little difference: coverage levels around $70 \%$ are already quite high, considering the proportion of small venues with few examiners. The standard deviation values obtained in all cases support the robustness of the results.

\subsection{Comparison With Experts' Solutions}

We will now assess the quality of the best and average GRASP solutions compared to the solution provided each year by human experts. Table 2 shows for all cases the objective values of expertise coverage $(A C)$ and total cost $(T C)$, as well as a measure of the degree of constraint satisfaction, defined as follows:

$C_{3}$ : Percentage of venues $v \in V$ where $\frac{|\operatorname{UBoard}(v)|}{|\operatorname{Board}(v)|} \in[0.4,0.6]$.

$C_{4}$ : Percentage of venues $v \in V$ where for every subject $s \in \operatorname{Lsubj}(v) \mid\{e \in$ $\operatorname{Board}(v): s=\operatorname{Subj}(e)\} \mid \leq M(v, s)$.

$C_{5}$ : Percentage of venues $v \in V$ where the number of education supervisors does not follow a uniform distribution.

Unlike the experts' solutions, both the best and average solutions provided by the GRASP algorithm fully satisfy constraints in all cases. At the same time, they improve in expertise coverage and costs, despite the fact that constraint satisfaction is usually obtained at the cost of worsening objective function values. Furthermore, comparing both Tables 1 and 2 we can see that even SGRC+SR improves the experts' coverage whilst simultaneously satisfying $C_{3}$ and $C_{5}$.

The solution provided by the proposed GRASP algorithm has another advantage: a considerable saving in working hours and effort for the team in charge of finding the EBA at hand. The differences between instances of the EBA problem made it impossible for the experts to somehow reuse previous solutions, so solutions had to be built from scratch, requiring full-time commitment from a team of experts for days. Time is specially critical because the input data are usually available only a few days before the UEE takes place. Our GRASP proposal allows to obtain a better solution with considerable less effort in a much shorter time. Additionally, the modular design of the algorithm allows it to be used as a replanning procedure in the case of unexpected incidences. It is also 
possible to perform some of the phases at hand, should it be the wish of the experts, or simply start from a human-made assignment and improve it. These characteristics add to the value of the proposed solution. Finally, it must be highlighted that, as from 2012, our method has substituted the human experts who can now employ their time and effort in more fruitful tasks.

\section{Conclusions}

We have tackled a real-world problem which consists in assigning a set of examiners to different boards in order to maximise expertise coverage in each board and also minimise the total costs incurred, subject to a series of hard and soft constraints. We have modelled this problem in the framework of multiobjective combinatorial optimisation with constraints and proposed a solving method, based on GRASP techniques, which significatively improves the results obtained by human experts: not only does it obtain better objective values while satisfying more constraints, but it has also proved a considerable saving both in time and effort for the team in charge of solving the problem.

\section{Acknowledgments}

This research has been supported by the Spanish Government under research grants FEDER TIN2010-20976-C02-02, MTM2010-16051 and MTM2011-22993.

\section{References}

1. Ehrgott, M., Gandibleux, X.: A survey and annotated bibliography of multiobjective combinatorial optimization. OR Spektrum 22 (2000) 425-460

2. Bouveret, S., Lemaître, M.: Computing leximin-optimal solutions in constraint networks. Artificial Intelligence 173 (2009) 343-364

3. Ernst, A.T., Jiang, H., Krishnamoorthy, M., D, S.: Staff scheduling and rostering: A review of applications, methods and models. European Journal of Operational Research 153 (2004) 3-27

4. Van den Bergh, J., Beliën, J., De Bruecker, P., Demeulemeester, E., De Boeck, L.: Personnel scheduling: A literature review. European Journal of Operational Research 226 (2013) 367-385

5. Resende, M.G.C., Ribeiro, C.C.: Greedy randomized adaptive search procedures advances and applications. In Gendreau, M., Potvin, J., eds.: Handbook of Metaheuristics, 2nd edition. Springer (2010) 283-320

6. Ernst, A.T., Jiang, H., Krishnamoorthy, M., Owens, B., D, S.: An annotated bibliography of personnel scheduling and rostering. Annals of Operations Research 127 (2004) 21-144

7. Della Croce, F.: Generalized pairwise interchanges and machine scheduling. European Journal of Operational Research 83(2) (1995) 310-319

8. Talbi, E.G.: Metaheuristics. From Design to Implementation. Wiley (2009) 